\title{
Translation Mode of Book of Change from the Perspective of Communication Science
}

\author{
Yushan Zhao \\ School of Foreign Languages North China Electric Power \\ University \\ Beijing, China \\ zhaoyushan1963@163.com
}

\author{
Miao Wang \\ School of Foreign Languages North China Electric Power \\ University \\ Beijing, China \\ shimmer16@126.com
}

\author{
Lijun Li \\ School of Foreign Languages North China Electric Power \\ University \\ Beijing, China \\ llj0426@163.com
}

\begin{abstract}
Based on the communication science and the communication mode put forward by Bittner, this paper constructs the translation mode of Book of Change. It analyzes the influential elements, such as translator, message, target audience, common experience in the translation process mode. The purpose of the mode is to help translator to recognize the translation process of Book of Change, improve the quality, readability and acceptability of the translation versions to reach the good effect of spreading Chinese culture and provide some suggestions to translation of Chinese classics.
\end{abstract}

Keywords-communication science; Book of Change; translation process mode

\section{INTRODUCTION}

Book of Change is one of the most influential and popular classics in Chinese history with plenty of wisdom and magic. It represents a powerful metaphysical and symbolic system of different aspects of Chinese culture. The translation study of Book of Change has been done from the traditional perspectives of linguistics and culture. As an emerging discipline, communication science provides a broader perspective for translators. The advantage of applying the communication science to the translation research lies in the fact that a great many factors in the communication process that affect the quality of translation can be taken into consideration. As Chinese classics vary with distinct characteristics, no certain communication mode can fully applicable to the translation of Book ok Change. In theoretical study and practice, certain mode cannot be applied mechanically. So the translation process of Book ok Change needs to be built according to its specific characteristics. After introducing the communication science, communication process communication elements as well as the application of communication approach to translation, this paper tries to construct a translation process mode of Book of Change. The author hopes the combination of communication theory and study of translation of Book of Change can provide a relatively new research perspective to let translators find and solve the problems in the translation process.

\section{BOOK OF CHANGE}

Book of Change, as a divination handbook full of mysteries because of its particular form and unique structure, has great influence on the life of all aspects, from business and politics to personal relationships. It has been venerated for more than three thousand years as an oracle of fortune, a guide to success, and source of wisdom. Scholars in different research area have explored its value from different perspectives.

\section{A. The Structure of Book of Change}

Book of Change is composed of three parts: the 64 hexagrams, their names and the hexagram judgments which are named yao and the 384 appended judgments (or linear judgments which is the understanding of $Y i$ from ancients. The ancients combined the first and second parts as Yijing and the third part is Yizhuan (also known as Ten Wings). Jing can be considered as the text while Zhuan is the appendixes to explain the whole hexagrams. Each hexagram has a name, figure, position and their opening text called judgments describing the general idea of the hexagram as a whole, and the six individual lines refer to specific elements in the development of the central idea. A hexagram consists of six lines on top of each other with forms of either broken or unbroken lines. The broken line is named "Yin line", and the unbroken line is named "Yang line". The lines are numbered from bottom up. Thus the first line is at the bottom while the last line is on the top. The combination of three lines generates a trigram, and the line is usually called Yao. In Book of Change, there are eight kinds of trigrams, namely, Qian, Kun, Zhen, Xun, Kan, Li, Gen, and Dui which respectively symbolize the heaven, earth, thunder, wind, water, fire, mountain and river, and these trigrams construct the hexagrams. It can be seen that they are the basic elements of the great nature. Each hexagram is constituted of two trigrams: the upper trigram is the outer trigram and the lower one named the inner trigram. Through permutation and combination of the two from the eight 
trigrams, totally 64 hexagrams come into being, and the functions, content and philosophical connotation of each hexagram are certainly not simple sum of the two components, but they presents a lot more from different aspects.

\section{B. The Influence of Book of Change}

The culture of Book of Change is the foundation and source of the development of Chinese culture and has great and profound influence on politics, economy, culture and other field. Asian countries introduced Book of Change to push forward its social reform. The book also reached the western world enjoyed high reputation throughout the world. The great wisdom and unique charm of Book of Change has attracted many scholars in the academic area. There is no doubt that its inexhaustible value will be continually explored both at home and abroad.

\section{Language Characteristics of Book of Change}

Book of Change mainly presents in three levels: phonetic level, semantic level and stylistic level. The phonetic element is the most fundamental unit of a text. Therefore, the sound pattern has great significance to the translator showing musicality and beauty. The rhyme, assonance and alliteration are frequently used in Book of Change. The meaning is basic function of a language. Translators usually ignore the fact that the original meaning of the words in Book of Change is often replaced by their extended meaning. Besides, there are a lot of loan characters in the book as a special linguistic phenomenon in Chinese classics. Ancient people sometimes did not use the correct character but used a substitute that has same or similar pronunciation with the correct one. Therefore, loan characters bring great barriers to translators to understanding the source text. Furthermore, Chinese classics have less punctuation, which is the other barrier for readers to fully understand the source text. The structure of Book of Change can be the most extinguishing feature with rich artistry of its language and profound meaning. The most conspicuous stylistic features of Book of Change are conciseness and rhythmicity. Moreover, the mysterious color of this book makes it much harder to express by another language with the same style.

\section{Three English Versions of Book of Change}

In recent years, more and more scholars have paid heed to the application of modem scientific theories in their study and oriented their researches on Book of Change from various novel perspectives. Up to date, there have been nearly thirty influential versions of the book in diverse languages, Latin, English, French and German, etc. Among a deal of foreign language versions, the first authoritative English version titled as The Yi King (Legge's version in short), which was translated by James Legge, a missionary, published in 1882 by Oxford University Press. Therefore, the English translations of Book of Change in the later times always took Legge's version for references at home and abroad. The first English version of Book of Change (W/R's version in short) was published in China in 1993 by Shanghai Foreign Language Education Press, which was translated by Wang Rongpei and Ren Xiuhua. Compared with Legge's version, W/R's version is much more concise without any annotation or appendix, which ended the history of the English translation of Book of Change all by scholars abroad. The version of Wang Rongpei and Ren
Xiuhua represents the spirit of Book of Change much better than those versions done by foreign scholars. Therefore, W/R's version has much significance in the history of crosscultural communication between China and the West.

\section{THE COMMUNICATION SCIENCE}

Communication science has its influence on translation. By introducing and analyzing three different communication modes and their major elements, it can be seen that communication and translation has common essence and studying translation from the perspective of Communication science is feasible and necessary.

\section{A. Communication Science and Communication Mode from Bittner}

The term "communication" originated from two ancient Greek "com", which means attempting to establish a certain relationship, and "munus" which means effectiveness, product, benefit, service and etc. The combined meaning of the two roots is "share" and "common". It emphasizes the meaning of "interactive" and "indirection" and lay stress on the equal status and interaction of sender and receiver. Although the definition of communication varies from person to person, most of experts believe that communication is a process in which meaning is assigned to a 'message' behavior or event [1].

The establishment of communication science is determined by three main sources: the behavioral science based on the spirit of scientism and positivism; Information Science based on information theory, cybernetics and systems theory; the Journalism focusing on the news communication and its principles. The founder of Information Scientist Claude E. Shannon, an American telecommunication engineer, presented information theory in 1948 and proposed a fundamental mode of communication process, known as Shannon-Weaver mode. The mode shows that communication is just a static, one-way process without taking into account the subjective initiative of communicator and the reciprocal effect from internal and external environment to each communication elements. Bittner J. R improved Shannon's mode and pointed out that communication events involve the following: source, message, channel, receiver, feedback, effect and noise[2]. A sketch of communication process is showed as following:

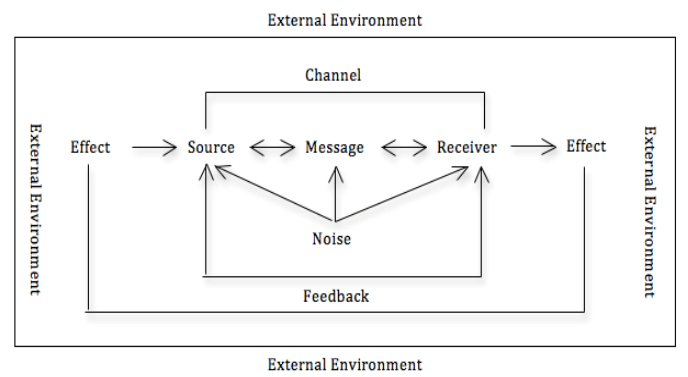

FIGURE I. COMMUNICATION MODE FROM BITTNER

In this communication mode, an information communicator, encodes ideas, opinions or other kind of information into message and sends them out to receiver by appropriate channel; a receiver decodes received message and 
then returns the reading effect to the source, the step is known as the "feedback". In the process of information transmission, the message will be interfered by external environmental factors and such interference is called "noise". The noise may interfere with the source, destination, or a direct impact the information in the communication process. In the improved communication mode by Bittner, the element of "feedback" made communication mode no longer a static and one-way process, but formed a more dynamic three-dimensional mode.

This paper selects the most classic and most suitable mode for this study to introduce and above description shows that the most basic mode is ordered, connected system and constituted by source, destination, message, feedback, noise and other factors. Meanwhile, the development and improvement of part of communication process, from singleline to dynamic three-dimensional and from simple to comprehensive are all reflected.

\section{B. The Influence of Communication Science on Translation}

The present translation study is characterized by lacking of a fully accepted theory, and Nida proposes that since translation is essentially a very complex phenomenon and insights concerning, the interlingual activity is derived from a number of different disciplines, e.g. linguistics, psychology, sociology, cultural anthropology, communication theory, literary criticism, aesthetics and semiotics, etc."[3] Nida explicitly suggests translation is communication, and presents its elements containing source, receiver, message, channel and noise and so on. Obviously, he borrows the terminologies and admits translation activity has the nature of communication science. In order to perfect its own knowledge, structure of theory, translation method, and solve a deal of controversial problems from some new perspectives, translation study borrows many theories from the other disciplines. Translation theorist Wolfram Wilss from Federal Republic of Germany thought previous research ignored many characteristics of translation itself, especially for the nature of information process. And he claims translating is a specific form of interlingual communication linked in linguistic act and decisions. The model "sender- to- receiver in communication process is ontologically equivalent to the model "source-language to target-language" used in translation theory. Interlingual translation is a special case in communication; its exceptional nature lies in the fact that two languages are involved in an act of linguistic communication, an SL and a TL [4].

\section{Application of Communication Appraoch to Translation}

As communication process and translation process share the same essence, that is showing message information processing and exchange, the major elements in the communication process also work in the translation process. In communication process, it mainly presents how the message reaches a receiver and which communication channel can achieve the purpose of communicators, while in translation process, it shows how a translator delivers the text message and the author's intention to the target readers, and guarantees those readers fully understand the message of source text and the intention of the original author. It can be seen that the communication mode and translation process has a lot in common, but translation process is more complicated, which still can be found sufficient theoretical support from communication science. Hereby based on communication approach, translation process will be analyzed through a detailed discussion of the major elements including: author, message, translator, target audience, feedback, and noise.

In the practical translation process, translators have a very active role to conduct their subjective initiative, which is known as "gatekeeper". The definition of message is the physical product that the source encodes and refers to the fact that communication has the responsibility of communicating message, exchange experience, inheriting culture of each cultural community and so on. Like the receiver in the communication studies, target receiver is an important factor that gets the message from the original author through the translator. Feedback means the receiver's response to the source's message, reflecting its communication effects. It actually shows a reversed communication process with which the original receiver turns to be the sender while the original sender becomes the receiver.

Besides the above elements in translation process which have no direct negative effect on the quality of translated text, there is another factor that is adverse impact on communication effect: noise. Noise is anything that blocks or interferes with the accurate message understanding and proper expression of original text of translators and correct understanding text and authors intentions of target readers. These are the theoretical support of applying communication mode to translation process and brief introduction of communication elements.

\section{TRANSLATION PRECESS MODE OF BOOK OF CHANGE}

Based on the communication mode and the major elements of translation process, the translation process mode of Book of Change is constructed as followed:

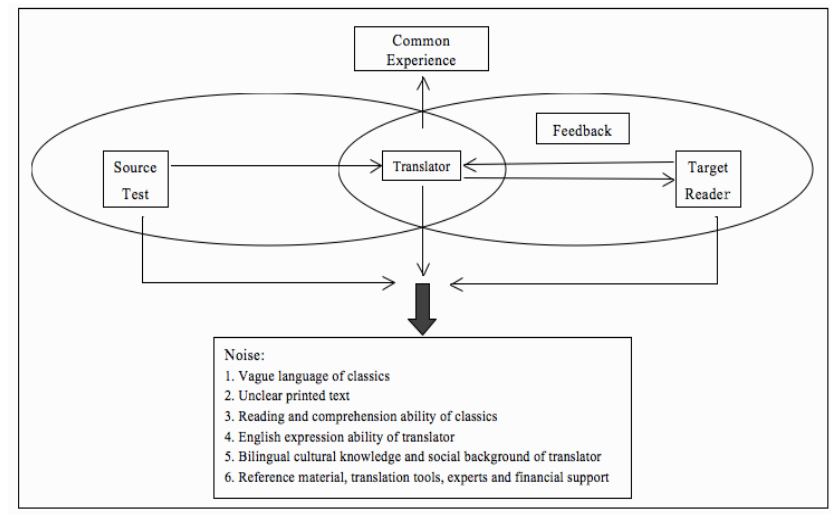

FIGURE II. Translation Process Mode of BOOK of ChangE

In the above translation process mode, the translator must decode the original text at first, after the full understanding of the original culture background, author's intention and even the philosophical implication, the translator transmits the reencoded translation to the target readers. And then the target readers will decode the translated version and give the feedback to the translator. Meanwhile, the internal and 
external noise during translation process can influence target readers' understanding of the original. From this translation process mode, it clearly shows that the translators stand at an absolute center position in the translation process. Avoiding noise and seeking common experience field are two critical elements related to the quality of the translation, while the feedback provided by target readers also plays an important role in improving the quality of translation.

In the translation process, the translator plays a dual identity, which means that the translator should not only understand the original, the corresponding historical and cultural background comprehensively and accurately, but also consider the cultural background of target readers to seek the intersection between two different languages and cultures. In addition, the translator must have profound language skills to pass the meaning of the original to the target readers with appropriate language and form.

Noise has relatively greater impact in this mode. At first, the noise comes from the text of classics itself, such as "Dao" is the core concept of Tao-Te Ching, and its connotation does not mean the same thing in different chapters and statements. Some refers to the origin of all things, some means the law, and while others are the truth of human behaviors. This kind of language ambiguity will bring translators more difficulties to understand the original. Besides, because some books were improperly stored or illegible printing, it is hard to read the original text for translators. Secondly, the noise also comes from the translators. The translator's ability to read Chinese classics, knowledge of history and culture and writing skills have direct impact on the quality of the translation. The other kind of noise is determined by the translator and readers' social environment, the translator's information, the use of advanced translation tools, the availability of adequate financial and social support, etc. Meanwhile, the social environment also influences the schedule and the translation quality of translation.

Due to the characteristics of the original, the role of common experience is more prominent in the translation process. Common experience refers to the knowledge domain for understanding during interpersonal communication. In the process of translation, it can be shown in the original text to authors and translators, and also shown in the translated text to translators and target readers. While the more intersection of the two kinds of common experience has, the easier the target readers can understand the translated version. From the language aspect, the translator should have flexibility in using bilingual languages. And especially in culture aspect, the translator should be familiar with the differences between languages in form, thinking mode and cultural connotation. It will help translators transplant the context of original text to the translated version in the classics translation process to achieve high quality translated text with exact meaning and the same style.

Feedback mostly presents in the target readers to the translated text. The positive feedback from target readers may encourage translators to participate in more translation work of classics books and improve the translation quality. While the negative feedback will help translators find problems, change translation strategies to improve their translation quality, and even participate in the translation updates and re-translation work.

From the above translation process model, we can see that translator should be initiative at first. Based on the principle of correct understanding, proper expression, and with the help of ancient Chinese experts or historians' research, translators need to take in-depth understanding of the relevant the explanation of classical allusions, annotations with modern Chinese, to correctly understand the original. Meanwhile, they should also practice and prefect their bilingual skills, and strive to complete the translated text vividly. In addition, translators should take the reading requirements, cultural level and other actual situation of target readers into consideration, and grasp the common experience with them to guarantee a greater communication results.

\section{CONCLUSION}

Book of Change is one of the oldest Chinese classics. It is a divination practice expounded with not only profound but also peculiar philosophical theory. Communication science is an effective theory to the research of Book of Change translation. Based on Laswell's Mode, Shannon-Weaver Mode and Bitter's Mode, the paper sums up the basic elements of general communication mode to apply to the translation process, such as author, message, translator, target audience, feedback and noise and constructs the translation process mode of Book of Change. The mode constructed is to help translators to understand the various stages and elements in the translation process, clarify the obstacles that may be encountered in the translation process, and solve the problem of the source text, cultural-loaded words and representation of original style. We suggest that the further study can be focused on some strategies and techniques for better-translated text of Book of Change according to major noises from the vague language of this book, common experience between two difference cultures with the help of the translation process mode of Book of Change.

\section{ACKNOWLEDGMENTS}

This research was supported by The Fundamental Research Funds for the Central Universities in 2014 named as "Research on Western Post-modernism and Translation” (No. 2014 ZD25) and The Fundamental Research Funds for the Central Universities in 2013 named as "Cognitive practice research on the application of metaphor and image schemas in lexical polysemy” (No. 13MS51) .

\section{REFERENCES}

S., Osborn, \& M.T., Motley, “Improving Communication”, New York: Houghton Mifflin Company, 1999, 4.

J. R., Bittner, “Mass Communication: An Introduction”, New Jersey: PrenticeHall, 1986.

E. A., Nida, "Language, Culture, and Translating”, Shanghai: Shanghai Foreign Language Education Press, 1993, p.110.

Wolfrom Wilss, "The Science of Translation-Problems and Methods", Shanghai: Shanghai Foreign Language Education Press, 2001, p.55.

FIGURE III. R. P. Wang, and X. H. Ren, "Book of Change", Shanghai: Shanghai Foreign Language Education Press, 1993. 